\title{
La importancia de la capacitación de las enfermeras del Hospital General de la Ciudad de Pachuca.
}

Miriam Hernández Juárez ${ }^{1}$ Juana Gabriela Islas Pineda ${ }^{2}$ Elizabeth Cruz Calva ${ }^{3}$

\section{Introducción}

Uno de los grandes temores del ser humano es la muerte. A lo largo de nuestra vida se nos enseña a vivir de tal o cual manera, pero nunca se nos enseña a morir. Morir es un proceso natural que forma parte del ciclo de la vida de los seres humanos. La muerte es un tránsito al descanso y a la vida eterna, claro que eta afirmación depende de la cultura y la creencia religiosa de cada persona.

Hablar de la muerte debe de ser algo muy natural pero a muchos de nosotros nos aterroriza el hecho de perder a un ser querido y más cuando se trata de un hijo. Este tipo de pérdidas son sumamente dolorosas para los padres, ya que siempre se piensa que los hijos ven morir a los seres que les dieron la vida.

Algunos casos que se presentan en distintos hospitales son decesos de recién nacidos; en este tipo de situaciones una gran parte del personal de Enfermería no sabe cómo actuar ante ello, pues el simple hecho de darle a conocer a los padres la noticia, les genera conflictos, pues no se sabe cómo abordar el tema y además se desconoce cómo ayudar a los padres para hacer menos dolorosa su pérdida.

Para esto es necesario capacitar al personal de Enfermería en cuanto a los cuidados tanatológicos postmortem para que puedan brindar servicios de mejor calidad a los padres que están pasando por este tipo de situaciones.
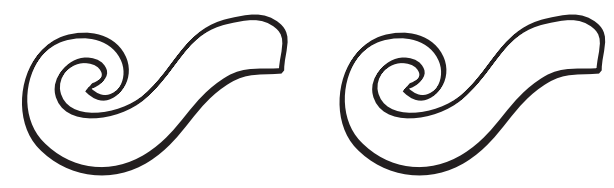

En el ámbito clínico, el profesional de Enfermería se encuentra con pacientes que pueden estar experimentando el duelo que se propicia por alguna pérdida de una persona allegada, tal es el caso de los mortinatos. El ser humano muere, en su mayoría, en instituciones de salud sin el apoyo de sus seres queridos y de servicios religiosos; aun cuando estén sus familiares, éstos no saben cómo sobresalir en el proceso de duelo al apoyar al paciente que transita por esta circunstancia. Todo lo enunciado exige atención de Enfermería tanto para el paciente como para sus familiares.

\section{Objetivos}

\section{Objetivo general}

Conocer las intervenciones del personal de Enfermería en los cuidados tanatológicos de mujeres con pérdida (mortinatos).

\section{Objetivos específicos}

Conocer el enfrentamiento y relación de enfermera-paciente y cómo interviene el personal de Enfermería en mortinatos.

\section{Preguntas de investigación}

¿Cómo intervenir de manera eficaz con los pacientes que tienen una pérdida como el mortinato?

¿Cuáles son los problemas más frecuentes por los que el personal de Enfermería no puede intervenir en el auxilio de pérdidas?

1 Octavo semestre de la Licenciatura en Enfermería. (andromeda artemisa@hotmail.com)

2 Octavo semestre de la Licenciatura en Enfermería. (gabyip7@hotmail.com)

3 Octavo semestre de la Licenciatura en Enfermería. (parís_ob@hotmail.com) 


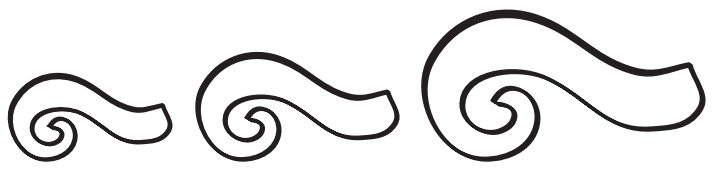

\section{Viabilidad}

A lo largo de esta investigación nos dimos cuenta que el tema abordado sigue siendo joven e inexplorado, de ahí el interés de ofrecer una opción que aminore la pérdida de seres amados.

Uno de los factores principales de viabilidad de nuestro trabajo fue que por medio de éste aumentará nuestro gusto por la investigación, no solo ese conocimiento nuevo adquirido quedará en nosotros sino también podrá difundirse a la sociedad, se podrá mostrar cómo se puede superar el duelo ante la pérdida de un ser amado.

\section{Justificación}

La finalidad de esta investigación es enfocarse en el cuidado tanatológico del paciente pediátrico y de la familia con la intención de hacer menos doloroso el fallecimiento de un bebé. $\mathrm{Al}$ parecer es poco el personal de enfermería que sabe acerca de los cuidados que se deben dar a los pacientes que así lo requieran, pues en muchos de los casos solo se hacen a un lado y dejan que la familia afronte su problema. Es importante ofrecer capacitación al personal de enfermería para que puedan crear un entorno de confianza y generen un clima de empatía que posibilite que las familias sientan apoyo y comprensión.

\section{Marco Teórico}

\section{Marco histórico}

"La primera unidad de cuidados paliativos de la que se tienen datos históricos fue en Roma en el año 474 D.C, fundada por Fabiola, la cual atendía a peregrinos procedentes de África". (Casarett, D./2005/p.p.73-88).

"Los originales hospice medievales no eran sitios en los que se cuidaba a los moribundos, sino lugares hospitalarios donde se recogían a

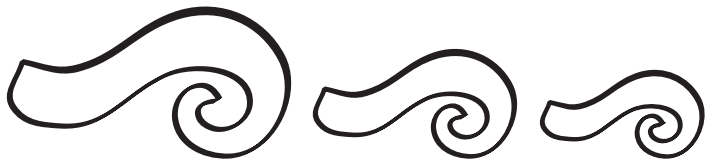

los forasteros y peregrinos para reponerse de las fatigas del viaje, comer, beber, dormir, cobijarse; pero algunos estaban tan enfermos o heridos, que incluso morían allí y quizás esto pueda explicar la relación con los hospice modernos. Jeanne Garnier es la primera en utilizar este nombre para referirse específicamente al cuidado de pacientes murientes; fundando en 1842 en Lyon el primer hospice o calvaire llamado Dames de Calvaire" (Saunders, C./2004/p.p.79-87).

"A finales del siglo XIX las Hermanas Irlandesas de la Caridad fundan el OurLady'sHospice en Dublín, dedicado exclusivamente al cuidado de pacientes incurables y murientes" (Tiberio, A. /2001/p.p.199-205).

"En 1905 se pone en marcha el StJoseph'sHospice de Londres, estos hospice son precursores de los actuales; eran verdaderas casas de la muerte en los que morían personas extremadamente pobres y donde se les dispensaban unos cuidados compasivos por caridad" (Callahan, D. /2000/p.p.440-448).

"La concepción del hospice moderno surge con Cicely Saunders en 1967 cuando fundó el St. Christopher's Hospice en Londres. Su apertura condujo a la apertura de diferentes sistemas de este tipo: centros de día, cuidados a domicilio, equipos de soporte" (Saunders, C. /2004/p.p.183-260).

"En EEUU se denominó Movimiento Hospice" (Mary Ann Liebert, Inc, USA, p. p.1127-1134).

La muerta ha acompañado al ser humano desde siempre. Hace muchos años no se tenían cuidados especializados para saber afrontar la pérdida de seres amados. Hoy en día, la labor de las enfermeras es de vital importancia en los momentos del fallecimiento de los bebés.

“En 1975, en Canadá, Balfour Mount fundó el Servicio de Cuidados Paliativos del Royal Victoria Hospital de Montreal y fue la primera vez 


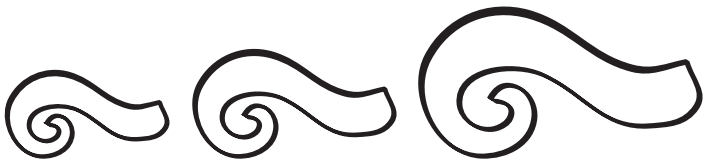

que se usó la palabra paliativo aplicado al cuidado tipo hospice" (Reyes, M.M., Nervi, F. \& Santi, B./2000/p.p.321-325).

“En Inglaterra, se reconoce la Medicina Paliativa con el rango de especialidad médica en 1987 y de enfermería" (Arranz, P., Barbero, J.J., Barreto, P.y Bayés, R./2003/p.p.294-298).

En 1990, la OMS unificó criterios hospiceo como cuidados paliativos y los define así:

"El cuidado global y activo de aquellos pacientes cuya enfermedad no responde a un método curativo y en los que es esencial el control del dolor y otros síntomas, la atención a los problemas psicológicos, sociales y espirituales, y el conseguir la mejor calidad de vida para el paciente y su familia" (Astudillo, E., Astudillo, W. y Mendinueta, C /1997/p.p.230235).

Recalca que: “Los cuidados Paliativos afirman la vida y consideran el morir como un proceso natural, no prolongan ni aceleran la muerte, proporcionan alivio del dolor y otros síntomas penosos, integran aspectos psicológicos y espirituales del cuidado, ofrecen un sistema de apoyo para ayudar a los pacientes a vivir tan activamente como sea posible hasta su muerte y a sus familiares para afrontar la enfermedad, la pérdida y el duelo" (Centeno, C./1997/p.p.1127-1134).

“El movimiento hospice llega a España de manos de Ruben Bild en el Hospital Universitario Marqués de Valdecilla de Santander en 1982 - 1984" (Doyle, D., Hanks, G., Cherny, N. \& Calman, K./2004/p.p.321-325).

“Jaime Sanz Ortiz pone en marcha la primera unidad de Cuidados Paliativos de España en 1985, incluida en la sección de Oncología de dicho hospital. En 1985, Ruben Bild desarrolla la primera experiencia hospice en Latinoamérica creando la Fundación Prager - Bild en Buenos Aires, con el lema Cuidar más allá de curar, cuyos consultores eran Robert G.

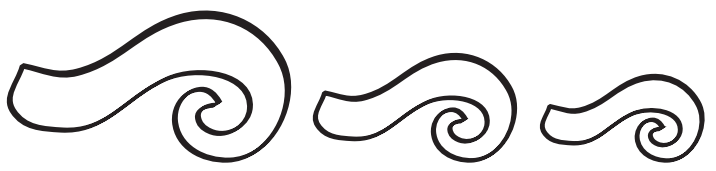

Twycross y Geoffrey Hanks, organiza el Primer encuentro internacional para el cuidado de las personas con enfermedad terminal en 1986, de la que fue Presidente de Honor Cicely Saunders" (Mount BM., Cohen /1993/p.p.161173).

"La implantación de los Cuidados Paliatios en España culmina en Cataluña con Xavier Gómez - Batiste y Joseph Porta, quienes crean las unidades de Cuidados Paliativos del Hospital de Sta. Creu de Vic en 1987 y la del Hospital de la Cruz Roja de Lleida ahora Arnau de Villanova en 1989". (Casarett, D. /2005/p.p.2229).

“En 1989 Marcos Gómez Sancho crea la Unidad de Cuidados Paliativos del Hospital El Sabinal de las Palmas de Gran Canaria" (LamontE./2005/p.p.205).

“En 1990 Juan Manuel Núñez Olarte pone en marcha la Unidad de Cuidados Paliativos del Hospital General Universitario Gregorio Marañón" (De Lima, L., Bruera, E./2000/p.p.12-21).

"En 1992 se funda la Sociedad Española de Cuidados Paliativos (SECPAL) en Madrid. En 1993 se recogen en una monografía del Ministerio de Sanidad y Consumo las primeras recomendaciones de la SECPAL en cuidados paliativos" Billings, A. (2000) Recent Advances Palliative Care en BMJ, Vol.321, p. 555-558.

Como se ha podido ver en el recorrido histórico anterior, los cuidados paliativos se han ido afinando y mejorando cada vez más en beneficio de las personas. Los cuidados continuos que se dan a los pacientes con enfermedad avanzada en los hospitales generales han llevado a disminuir el número de pacientes que necesitan ser admitidos en un hospital al final de sus vidas. En las últimas semanas de vida los pacientes y la familia tienen necesidades incrementadas, la importancia primaria es proveer información y facilitar la comunicación con referencia a la aparición de probables síntomas, para reducir su impacto en la familia y el paciente 


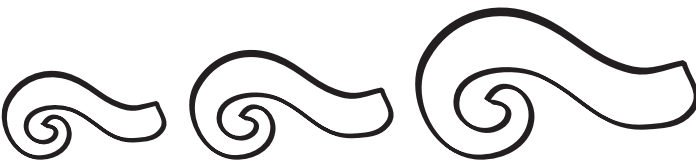

Marco conceptual

Tanatología

Para poder entender más acerca de este tema, es necesario saber que es la Tanatología; pero antes debemos de saber que para algunas personas la muerte es vista desde varios puntos de vista como el biológico, Ruy Pérez Tamayo nos dice:

"La muerte es un proceso que ocurre en los seres vivos; se inicia cuando los cambios son irreversibles; se caracteriza por la pérdida de la complejidad de su organización y por la disminución en el contenido de energía, y termina cuando la diferencia de este contenido energético con el medio ambiente es cero, todo es parte de un proceso biológico". (Pérez Tamayo, Ruy (2002); Acerca de la muerte; Bogotá, Colombia: Giro Editores; p.p. 22)

Algunos autores como José Carlos Fuentes Rocañin, ven a la Tanatología desde un punto de vista médico-legal y la definen como:

"La Tanatología es la parcela de las ciencias forenses que se encarga del estudio de todo aquello que tiene que ver con la muerte de la persona desde las cuestiones puramente administrativas hasta los detalles biológicos más especializados." (Fuentes Rocañin, José Carlos (2007); Manual de Ciencias Forenses; Madrid, España: Arán Editores; p.p.101)

Sin embargo, a mediados del siglo XX, la Tanatología fue considerada, no solo la ciencia que trata con los cadáveres y todo lo que tenga que ver con el proceso legal que esto conlleva, sino que para personas como la Dra. Elizabeth Kübler-Ross es considerada como:

"Es la ayuda otorgada a los enfermos en fase terminal a vivir gratamente, sin dolor y respetando sus exigencias médicas". (Kübler-Ross, Elizabeth (2011); Antología de Tanatología; Pachuca de Soto, Hidalgo; p.p. 15).

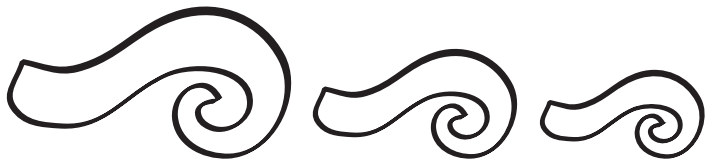

Sin embargo, desde nuestro punto de vista no solo se ayuda al enfermo terminal, sino que de alguna u otra forma se brinda apoyo a los familiares, no solo durante el proceso de enfermedad del paciente en fase terminal, sino que hay personas que súbitamente han perdido algún ser querido y requieren enfrentar el proceso de duelo para poder entender lo que pasa a su alrededor, concientizarlos de la nueva vida sin el ser amado y así poder llevar una vida más tranquila y encontrar un nuevo sentido de vida.

En el caso de la pérdida de un hijo, el daño causado no es fácil de entender, pues la mayoría de los seres humanos piensan que el ciclo natural de la vida es que los padres mueran antes que los hijos. Cuando un padre se enfrenta a la muerte de un hijo es un proceso devastador, pues no lo ven como un proceso normal.

\section{Mortinato}

Se refiere a la muere inmediatamente después del nacimiento, en este caso los mortinatos, que se definen como:

"Un feto que se esperaba que sobreviviera muere durante el nacimiento o durante la segunda mitad del embarazo". (Cunningham FG, Leveno KL, Bloom SL(2005); Medicina Obstetrica; México, D.F.: McGraw-Hill; p.p. 29) Los padres no saben cómo vivir su duelo, pues la sociedad les dice que la vida debe de seguir y que ya vendrán más hijos, esto les crea una confusión al respecto, pues es un hijo que no conocieron y de cierto modo no se "crearon" lazos tan fuertes como con alguien que convives a diario.

La mayor parte de los decesos ocurren en un hospital, pero realmente no sabemos si el personal cuenta con la capacidad para brindar ese apoyo humano y moral que la familia necesita al momento en que ocurre la pérdida, como ya mencionamos antes, la Tanatología no solo se enfoca en la parte médico-legal; parte en la cual consideramos que tampoco están del todo preparados; sino que también se brinda apoyo a aquel que necesite enfrentar un duelo. 


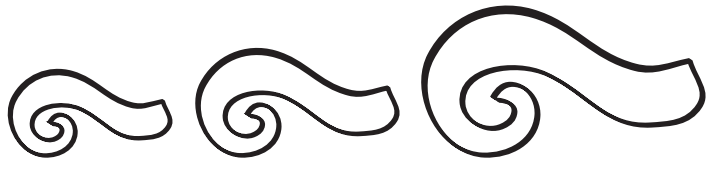

Marco teórico

"Todo el mundo experimenta muchas pérdidas a lo largo de la vida, pero la muerte de un ser querido no tiene comparación por el vacío y la profunda tristeza que produce". Elizabeth Kubler Ross y David Kessler (2006)

La enfermera en el transcurso de los tiempos se ha caracterizado por su gran humildad y humanidad, por minimizar el dolor físico y espiritual de los seres humanos.

Dedicada al bienestar total del enfermero, prolonga el arte de la medicina y enaltece la bondad de su profesión.

Es la mano amiga que ayuda al ser doliente, que le acompaña en su caminar durante el sufrimiento y la enfermedad.

El no abandonar a un enfermo es un compromiso ético que consiste en acompañarlo en el trayecto de la enfermedad sin importar cuál sea su desenlace; sería muy gratificante que al final de la vida, justo al emprender la partida, el moribundo pueda tener una enfermera amiga con gran calidad humana, que le permita morir con su compañía, aliviándole sus síntomas y disipando sus temores.

\section{Enfermería en la tanatología}

La muerte no es un hecho o un instante, es un proceso que inicia desde el momento de nuestra concepción. Es un misterio inexplorado lleno de incógnitas, temores, oportunidades, angustias, opciones y es inherente a la vida, va de la mano en cada instante de nuestro largo caminar.

En 1858 Florence Nightingale escribió que el objetivo fundamental como enfermeras es "situar al paciente en el mejor estado posible para que la naturaleza actué en él". La profesión de enfermería es una ciencia pero también es un arte. (González Castro María del Carmen, 2007).

Elisabeth Kubler- Ross pionera del movimiento de cuidados paliativos en el mundo entero, fue la primera profesional que se acercó deci-

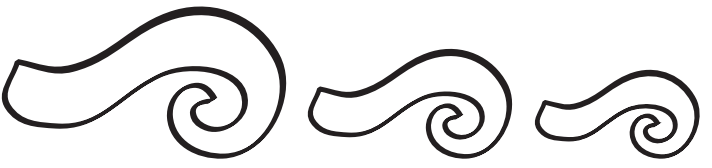

didamente a los enfermos que iban a morir y a sus problemas. Dedicándose completamente a pacientes terminales, acompañándolos y observando cada uno de sus aspectos y relacionándose con ellos para tratar de aceptar de una manera positiva y humana su condición.

La doctora Kübler en su libro La muerte, un amanecer, nos habla de todas las condiciones en la que se presenta un individuo. También habla de la muerte y la forma de vivirla en ese momento, esta experiencia es general e independiente del hecho de que la persona sea un aborigen de Australia, un hindú, un musulmán, algún creyente o un ateo, por ejemplo. Es independiente también la edad o el nivel socioeconómico, puesto que se trata de un acontecimiento puramente humano, de la misma manera que lo es el proceso natural de un nacimiento.

Esta autora hace referencia y similitud de la muerte con un mariposa, es precisamente con este ejemplo que les hablaba a los niños moribundos de muy corta edad, mencionaba que la muerte física del hombre es idéntica al abandono del capullo de seda de mariposa: "Morir significa, simplemente, mudarse a una casa más bella".

La muerte física del hombre es idéntica al abandono del capullo de seda por la mariposa. La observación que se hace, es que el capullo de seda y su larva pueden compararse con el cuerpo humano. Desde el momento en que el capullo de seda se deteriora irreversiblemente, ya sea como consecuencia de un suicidio, de homicidio, infarto o enfermedades crónicas (no importa la forma), va a liberar a la mariposa, es decir, a nuestra alma.

\section{Metodología}

\section{Enfoque}

Por medio de este trabajo podremos demostrar la importancia de que el personal de enfermería tenga conocimientos en cuidados paliativos hacia cualquier tipo de pacientes y pueda aplicarlos en momentos oportunos. 


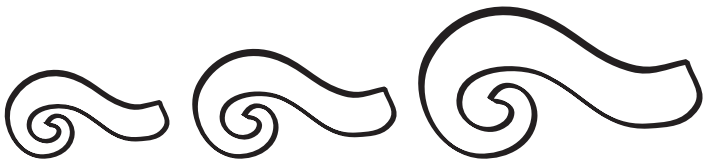

De acuerdo con nuestras expectativas podremos realizar campañas informativas al público en general para cuando enfrente situaciones de pérdida. Sin embargo, le estaremos demandando y exigiendo al sector salud que brinde capacitación a su personal para ofrecer un mejor servicio a las familias. De igual for$\mathrm{ma}$, se le puede sugerir a este sector que instale centros de ayuda que contribuirán a una mejor calidad de vida para la sociedad.

\section{Alcance}

Nuestro tema pertenece al tipo descriptivo. Por medio de él estamos detallando un hecho en un contexto social; buscando especificar las propiedades más importantes y esenciales. En esta investigación se hizo uso de la metodología tanto cuantitativa como cualitativa con el objetivo de obtener un trabajo más completo y objetivo.

Un alcance notable de este trabajo fue hacerles ver a las enfermeras que la capacitación es imprescindible para que se desempeñen de mejor forma en su trabajo.

\section{Diseño}

Este trabajo tiene un diseño no experimental pues no se manipularon deliberadamente las variables. Se trató de observar el fenómeno tal y como se dio en su contexto natural, para después analizarlo. Se hicieron inferencias sin la intervención directa sobre la variación de las variables. Es del tipo transeccional ya se estudió en un momento dado.

\section{Hipótesis}

- Las enfermeras del Hospital General de la ciudad de Pachuca, desconocen cómo actuar con los familiares que pierden a bebés recién nacidos

- Los padres de familia que pierden a sus bebés desconocen de los cuidados o servicios que les pueden brindar las enfermeras.

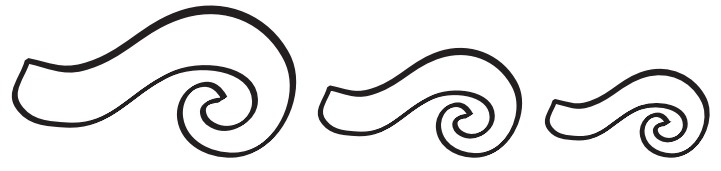

\section{Selección de la muestra}

SUJETO: Personal de enfermería en el área de neonatología en cuidados intensivos del Hospital General de Pachuca, Hgo.

POBLACIÓN: En cada turno hay 24 enfermeras, hay un total de 72 .

MUESTRA: 40 enfermeras(os).

\section{Procedimiento}

\begin{tabular}{|c|c|c|c|c|c|c|c|c|}
\hline Actividades & 13 & 14 & 15 & 25 & 26 & 27 & 28 & 29 \\
\hline Solicitar oficio & & & & & & & & \\
\hline $\begin{array}{c}\text { Realizar cita con } \\
\text { coordinandora de } \\
\text { ensenananza }\end{array}$ & & & & & & & \\
\hline $\begin{array}{c}\text { Avisar al } \\
\text { personal de } \\
\text { enfermeria }\end{array}$ & & & & & & & \\
\hline $\begin{array}{c}\text { Aplicación de } \\
\text { instrumentos }\end{array}$ & & & & & & & & \\
\hline
\end{tabular}

\section{Resultados}

Con respecto a los cuidados tanatológicos para el paciente neonatal, la mayoría del personal de enfermería al que se le aplicó las encuestas, coinciden en que no solo la especialista puede atender este tipo de situaciones, pues en ocasiones ellas se han visto en la necesidad de ofrecer este tipo de servicios sin ser enfermeras especialistas. Pocas fueron las personas que no saben quién debe de otorgar esta atención, pues creen que ambas pueden realizarlo. $\mathrm{Al}$ parecer ninguna enfermera considera prudente que este tipo de cuidados sean otorgados por auxiliares, pues les falta mucha experiencia y preparación en cuanto a este tema.

La gran mayoría del personal coincide en que los cuidados paliativos son otorgados a los pacientes neonatales, pues opinan que todos tenemos derecho a un trato digno y no por el simple hecho de que estén en estado agónico se les va a menospreciar. Otro porcentaje indica que no se llevan a cabo, pues muchas veces no saben cómo acercarse a los padres ni mucho menos cómo abordar el tema de esta 


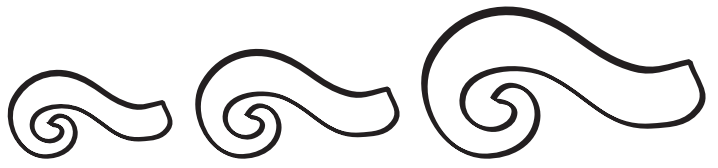

pérdida tan significativa. Algunas otras enfermeras mencionaron que ya no es necesario aplicar estos cuidados y otras que no se aplican para nada.

En lo que concierne a la unidad de dolor, la mayoría del personal entrevistado considera que no es necesaria una unidad del dolor, pues en la medida que pueden tratan de orientar y ayudar a los pacientes que presentan casos como estos. Otros tantos desconocen o afirman que una unidad de dolor está en el hospital, lo cual es cierto, existe pero pocos hacen uso de ella por falta de orientación e información.

El personal de enfermería coincide en que el servicio de atención psicológica que es brindado a los padres es solo cuando ellos lo solicitan, pues otros muchos casos desconocen de la ayuda o el personal no sugiere este tipo de atención por pena o no saben cómo plantearle a la familia este tipo de ayuda. Un porcentaje bajo del personal entrevistado sugiere que no es necesaria la atención.

Con respecto a otorgar y brindar el servicio psicológico a los padres antes y después de una pérdida significativa; obtuvimos que el hospital cuenta con una excelente plantilla de trabajo, con personal capacitado y con calidad en su ramo; por lo que sería conveniente que los jefes del servicio hicieran hincapié en abrir una área específica para este problema, ya que de alguna manera podemos evitar otras dificultades relacionadas con el que presentan o simplemente lo podemos canalizar y remediar a tiempo. Ser un hospital de vanguardia con lo que va requiriendo nuestro paciente día con día. Con la satisfacción total de cada una de sus necesidades.

Con base en los resultados obtenidos se puede afirmar que el hospital cuenta con un personal preocupado por brindar un servicio de calidad y calidez, se ha preparado para ser aún

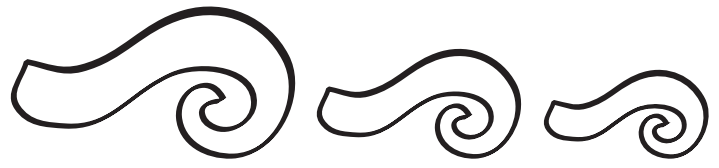

más eficiente con lo que va necesitando tanto su paciente, como sus familiares. En 1858 Florence Nightingale escribió, que el objetivo fundamental como enfermeras es "situar al paciente en el mejor estado posible para que la naturaleza actué en él". Como ya se mencionó con anterioridad, la profesión de enfermería es una ciencia, pero también es un arte.

No es necesario que el personal de enfermería sea sometido a cursos de capacitación ya que cuentan con ella. Se sugiere que se continúe con la actualización del personal. Se pueden dividir los temas de actualización en dos: información sobre nuestro sector de actividad y nuevas herramientas de trabajo. Información sobre nuestro sector de actividad incluye: quiénes son mis competidores, potenciales externos, crecimiento del sector versus crecimiento de mi persona, entre otras. En nuevas herramientas de trabajo, se pueden mencionar los siguientes: liderazgo, trabajo en equipo, sistema de calidad, delegación y mandos medios, entre otras.

El presente es complejo, la competencia es cada vez más importante y diversa, y los pacientes requieren cada vez más sobre nosotros. Ellos desean tener confianza en el personal de enfermería.

La información constante y actualizada debe incluir las certezas y las dudas que van suscitando las diversas posibilidades diagnósticas y terapéuticas. Es útil que el paciente y su familia sean invitados a comentar en sus propias palabras la información recibida. En más de una ocasión nos sorprenderá la enorme diferencia que existe entre aquello que habíamos querido decir y aquello que fue interpretado. En definitiva, el manejo de la información al paciente, más allá de una técnica, responde siempre a una ideología. La nuestra puede sintetizarse en cuatro conceptos básicos: veracidad, confiabilidad, estimulación de confianza y evitar la agresión. 


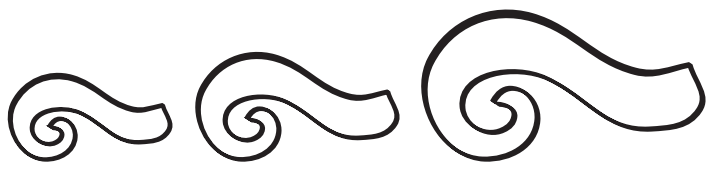

Conforme al mantenimiento de la información sobre los cuidados paliativos, el profesional de enfermería se presenta en un porcentaje mayor, debido a que es el que se encarga de dar un enfoque especial al cuidado de los pacientes y por el simple hecho de tener un contacto superior, cabe mencionar que a pesar que enfermería es sin duda el principal personal que da este tipo de atención, los demás profesionales de salud en este caso los médicos participan activamente para brindar este tipo de información.

Como podemos observar, el mayor porcentaje cayó en la respuesta "no", esto demuestra que le es fácil al profesional de enfermería aplicar los cuidados tanatológicos.

La frecuencia de la tipología de pacientes el Hospital General de Pachuca en el grado de cuidados paliativos con calidad de mortinatos es ocasional, en su mayoría, pero bebemos decir que en servicios especializados como pediatría se encuentran en mayor número.

\section{Conclusión}

De acuerdo con los resultados obtenidos no es necesario capacitar de una manera profunda al personal de enfermería ya que cuenta con los principios básicos de qué hacer en casos como estos, pues como mencionaban algunas enfermeras, nadie es inmune al dolor y como ser humano, en muchas ocasiones se ayuda por instinto y más cuando se trata de pérdidas tan significativas en la vida de los padres. Lo que sí sería factible es mantener informados a los padres de familia de cada procedimiento y evolución de sus pacientes para que no existan dudas durante el transcurso de su estadía.

En verdad lo que nos gustaría es la implementación de una clínica del dolor, apoyo psicológico a los familiares, ante cualquier circunstancia por la que esté pasando en ese instante. Como seres humanos dedicados a la salud tenemos el deber y la obligación de estar en equilibrio bio-psico-social y espiritual para

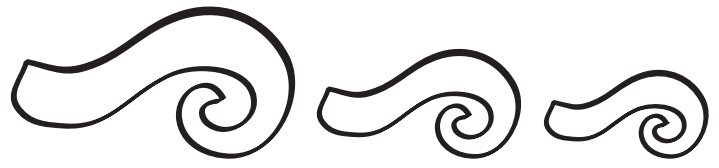

mantener un estado de salud pleno y poder dar una atención completa a nuestros enfermos. Aquí estaríamos colaborando de manera conjunta lograr dicha construcción, tanto del personal como de pacientes y familiares y por tanto demostrar lo importante que es el tenerla.

Realmente supimos con exactitud que mucho del personal que se encuentra trabajando en el Hospital General de la ciudad de Pachuca no cuenta con la capacitación necesaria para atender a este tipo de pacientes o que la información con la que cuentan es insuficiente o no actúan adecuadamente, pues no saben cómo abordar a los familiares de este tipo de pacientes.

Se considera que lo mejor sería que en este tipo de instituciones se orientara de una mejor manera al personal de enfermería para que puedan contribuir a dar un mejor servicio en cuanto a cuidados de tanatología, pues como ya lo hemos mencionado anteriormente, muchas o muchos de ellos no saben qué hacer en estos casos y piensan que lo mejor es dejarlo pasar y tienen la creencia de que entre menos intervención tengan será lo mejor para los padres de familia. Esto no solo les ayudaría a tratar a los pacientes en calidad de mortinato, sino que a la familia se le brinda mayor confianza y con ello se les ayuda a sobrellevar su pesar.

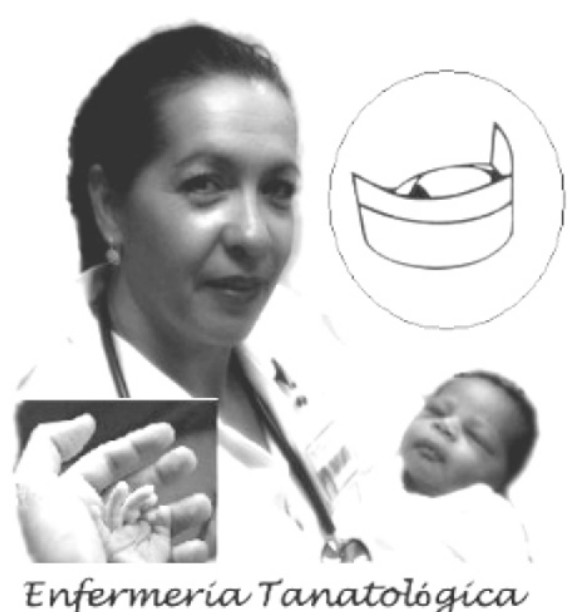



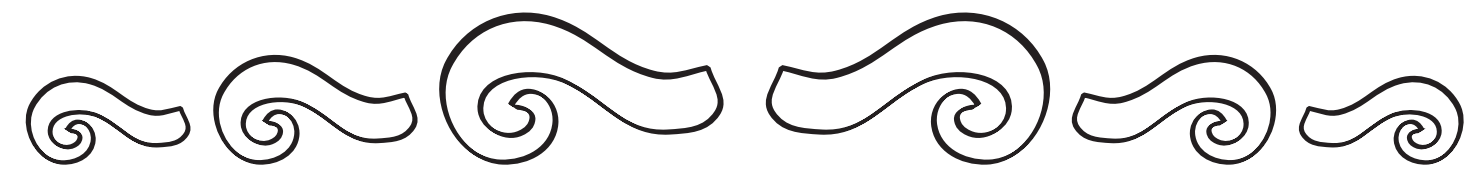

\section{ANEXOS}

\section{Paciente neonatal}

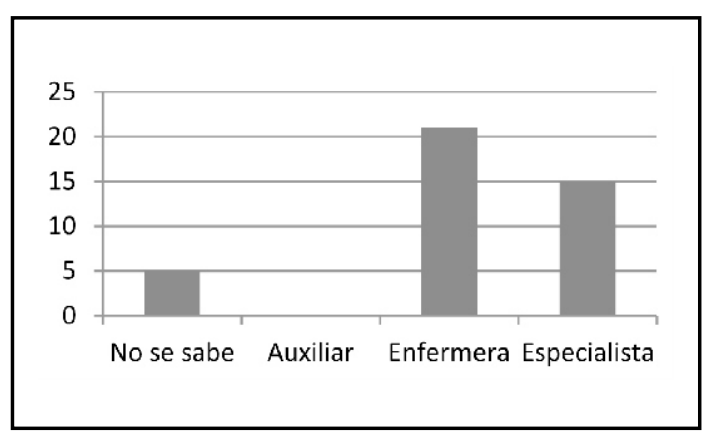

\section{Unidad de dolor}

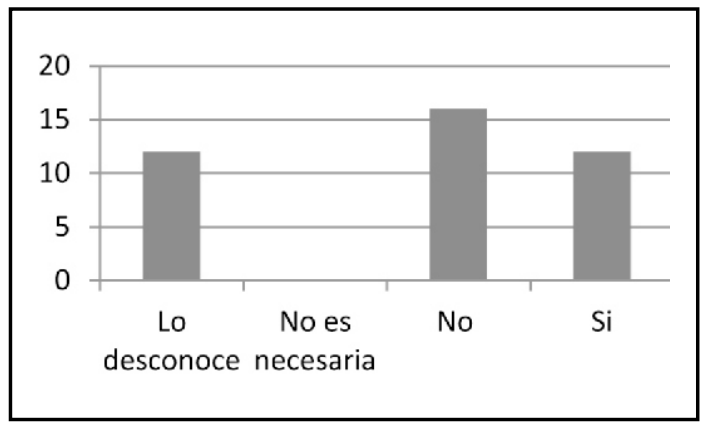

\section{Servicio psicológico}

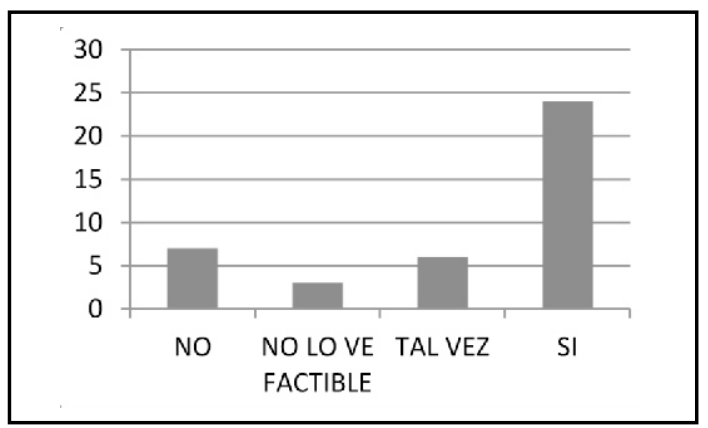

\section{Cuidados paliativos}

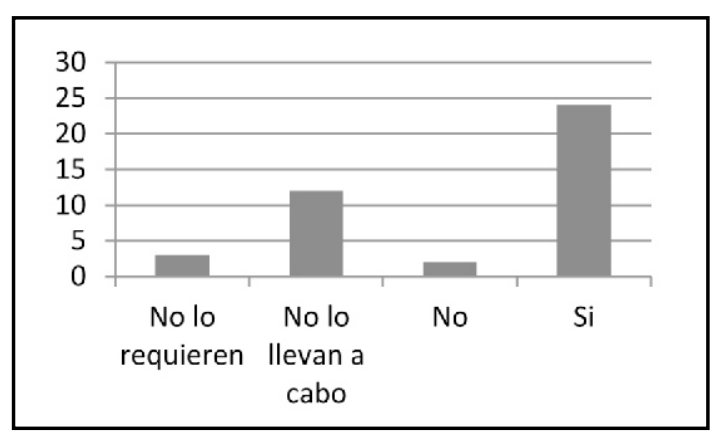

4. Atención psicológica en pérdida significativa

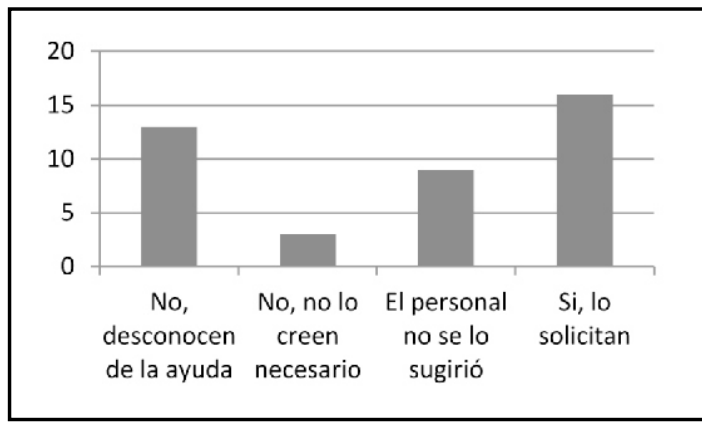

6. Capacitación del personal

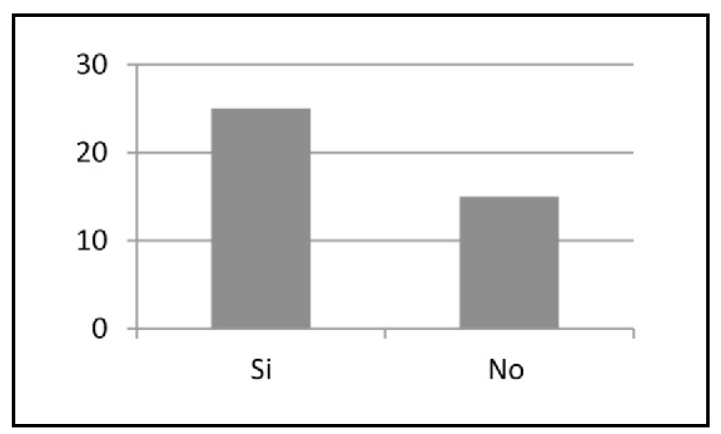




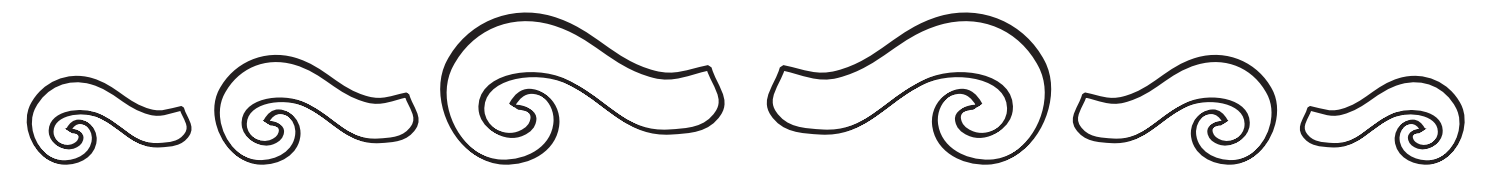

\section{Aplicación de cuidados}

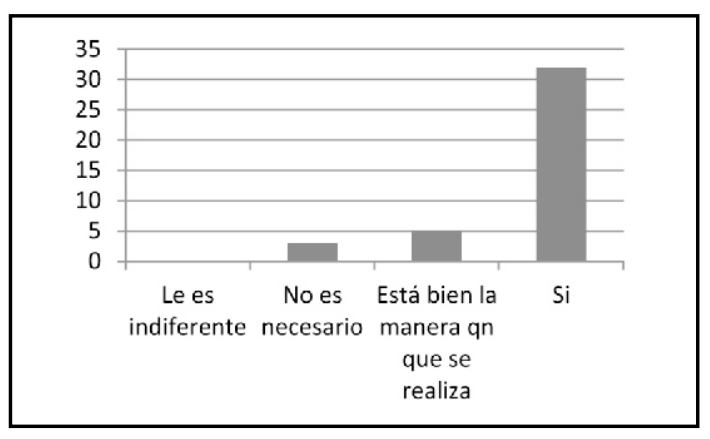

\section{Información sobre cuidados paliativos}

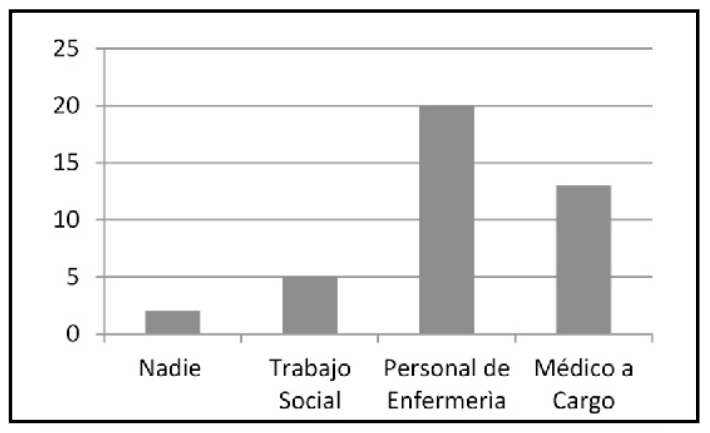

\section{Padres informados}

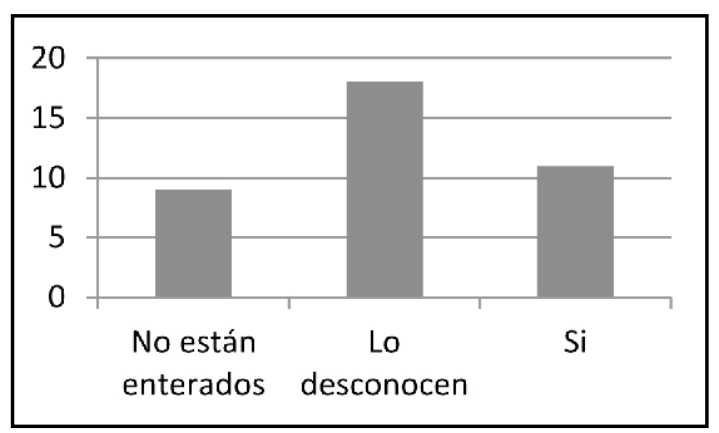

\section{Problemas legales}

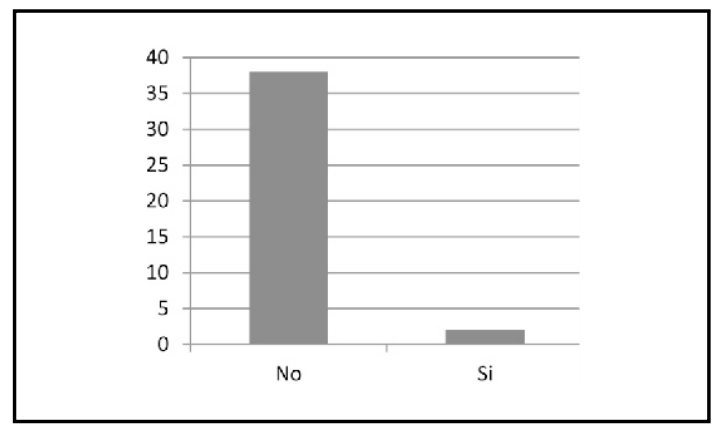




\section{Fuentes de consulta:}

KOZIER BARBARA, ErbGlenora, Berman Audrey, SnyderShilee, (2005), Fundamentos de Enfermería, Madrid, Mc Graw-Hill.

ROSALES BARRERA, Susana, Reyes Gómez Eva, (2007) Fundamentos de Enfermería, México, D.F., Manual Moderno.

PÉREZTAMAYO, Ruy;(2002) Acerca de la muerte. Bogotá, Colombia: Giro Editores.

FUENTES ROCAÑIN, José Carlos;(2007) Manual de Ciencias Forenses; Madrid, España: Arán Editores.

KÜBLER-ROSS, Elizabeth; (2011) Antología de Tanatología; Pachuca de Soto, Hidalgo.

CUNNINGHAM FG, Leveno KL, Bloom SL;(2005)Medicina Obstetrica; México, D.F.: McGraw-Hill.

Cuidados paliativos, Filosofía de los cuidados paliativos, http://www.elergonomista.com/enfermeria/en06.html,consultado en marzo de 2011.

Cuidados del personal de Enfermería, Cuidados paliativos, http://escuela.med.puc.cl/publ/boletin/20071/CuidadosPaliativs.pdfconsultado en mayo de 2011.

Afrontamientos ante la pérdida, Duelo, http://www.fmca.org.mx/art/art.php?id $=1300_{\text {, }}$ consultado en abril de 2011.
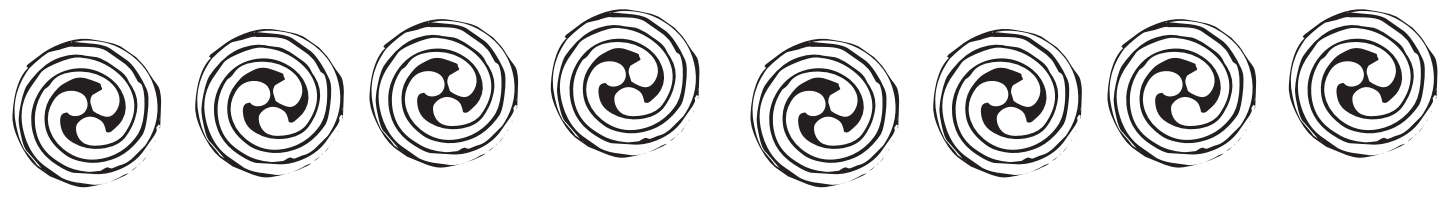

๑15@ 


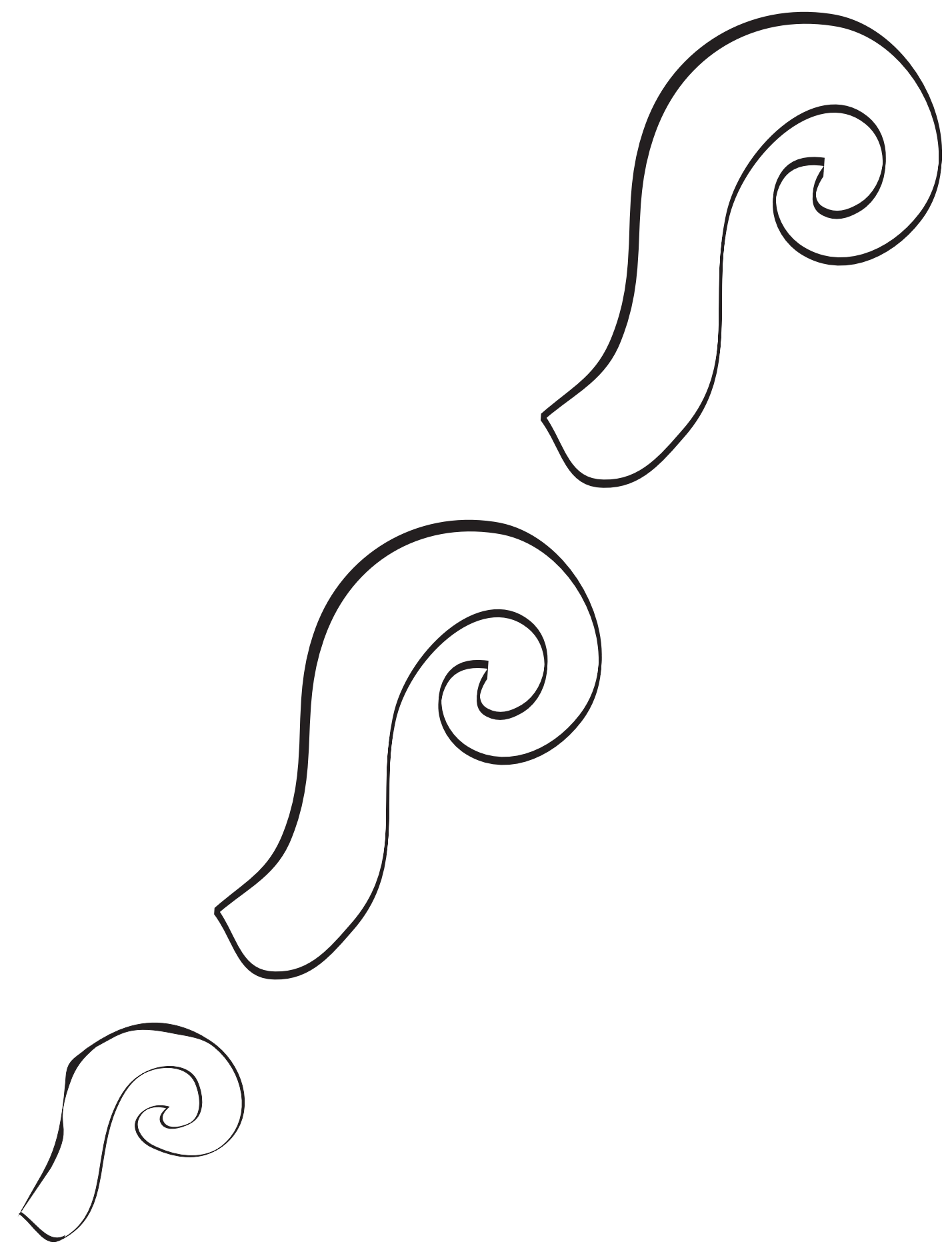

216@ 